ORIGINAL PROF-2237

\title{
TYPE 2 DIABETES MELLITUS;
}

FREQUENCY OF HYPOMAGNESEMIA IN PATIENTS

Dr. Raheel Iftikhar, Dr. Sultan Mehmood Kamran, Dr. Kumail Abbas, Dr. Ehtesham Haider

ABSTRACT... Objective: To determine frequency of Hypomagnesaemia in patients of type 2 diabetes mellitus in our population. Data Source: Random selection of DM II patients from Outpatient Department CMH, Kharian. Design: Cross sectional study. Setting: Combined Military Hospital Kharian, Department of Medicine. Duration of study: January 2011 to December 2011. Materials \& Methods: We selected outdoor patients of DM-2 from both gender between 40 to $70 \mathrm{yrs}$ of age by random sampling. Those selected, were subjected to blood fasting and random glucose measurements as well as serum magnesium levels. Blood samples were collected using full aseptic measures and within one hour, samples were transported to Armed Forces institute of Pathology (AFIP) for analysis. Serum magnesium level estimation was done by timed endpoint method using calmagite dye. DXC 600 automated analyzer was used. The results were verified by Pathologist. Results: The overall frequency of Hypomagnesemia was $32.2 \%$ (124 out of the 385 subjects) using the cutoff value of less than $0.6 \mathrm{mmol} / \mathrm{l}$ for Hypomagnesaemia, whereas $67.8 \%$ (261 out of the 385 individual) had normal serum magnesium levels. Conclusions: Significant number of patients with type 2 diabetes mellitus suffers from Hypomagnesaemia. These patients have increased risk risk of poor Glycemic control and diabetic complications due to Hypomagnesemia. Therefore, it is recommended that serum magnesium levels should be checked regularly in patients with type 2 diabetes mellitus and oral magnesium replacement should be done.

Key words: Diabetes Mellitus, Hypomagnesaemia, insulin resistance, diabetes complications.

Article Citation

Iftikhar R, Kamran SM, Abbas K, Haider E. Type 2 Diabetes Mellitus; Frequency of Hypomagnesemia in patients. with . Professional Med J 2013;20(5): 804-809.

\section{INTRODUCTION}

Magnesium is the fourth most abundant cation in the human body and the second most abundant intracellular cation'. It can exist naturally in three forms. It may be protein-bound, complexed, or free cation. It serves as a co-factor for all enzymatic reactions that require ATP and as a key component in various reactions that require kinases ${ }^{2}$. It is also an essential enzyme activator for neuromuscular excitability and cell permeability, a regulator of ion channels and mitochondrial function, a critical element in cellular proliferation and apoptosis, and an important factor in both cellular and humoral immune reactions. Magnesium deficiency has been demonstrated in $2.5-14 \%$ of hospitalized patients and is found to coexist in up to $40 \%$ of patients with other electrolyte abnormalities, particularly hypokalemia and to a lesser extent, hyponatremia or hypocalcaemia ${ }^{3}$. Magnesium $(\mathrm{Mg})$ serves as is a cofactor in both glucose transporting mechanism of cell membranes and various enzymes important in carbohydrate oxidation ${ }^{4}$. Different endocrine and metabolic disorders are associated with magnesium deficiency. These include diabetes mellitus, Cushing syndrome and hypothyroidism ${ }^{5}$. Approximately 90 to $95 \%$ of all diagnosed cases of diabetes mellitus (DM) have type 2 diabetes mellitus (DM) ${ }^{6}$. Diabetes mellitus was reported to be the sixth leading cause of death listed on US death certificates in $2007^{7}$. About 6.9 million people are suffering from diabetes mellitus in Pakistan $^{8}$. Diabetes mellitus has been suggested to be the most common metabolic disorder associated with magnesium deficiency, having 13.5 to $47.7 \%$ prevalence $e^{3,4,9}$. Hypomagnesemia in DM represents secondary magnesium depletion which requires more or less specific correction of the different perturbations of the control mechanisms of magnesium deficit that are involved with diabetes. Osmotic diuresis clearly accounts for a portion of the magnesium loss ${ }^{6}$. On one hand Hypomagnesemia is associated with type 2 diabetes and on other hand numerous studies have reported an inverse 
relationship between Glycemic control and serum $\mathrm{Mg}$ levels $\mathrm{s}^{3.5}$. Although many authors have suggested that diabetes per se may induce Hypomagnesemia, others have reported that higher Mg intake may confer a lower risk for type 2 diabetes ${ }^{4}$. Different studies have shown that those diabetics who have low serum magnesium levels have a poor Glycemic control, increased insulin resistance and rapid progression to diabetic micro and macro complications ${ }^{3-4,7,9}$. It has been suggested that Hypomagnesemia may induce altered cellular glucose transport, reduced pancreatic insulin secretion, defective post receptor insulin signaling, and/or altered insulin-insulin receptor interactions ${ }^{10}$. In patients with type 2 diabetes, oral $\mathrm{Mg}$ supplementation during a 16-wk period was suggested to improve insulin sensitivity and metabolic control ${ }^{11}$. This study will help to find out frequency of Hypomagnesemia in diabetic population in Pakistan and can provide guide to start magnesium replacement in diabetics and pre-diabetics to help improve Glycemic control and to reduce rate of progression to diabetic complications.

\section{METHODS \& MATERIALS}

385 patients of type 2 DM were selected randomly from Outpatient Department of Medicine, $\mathrm{CMH}$ Kharian (a tertiary care hospital) in 01 year from January 2011 to December 2011. Those selected, were subjected to blood fasting and random glucose measurements as well as serum magnesium levels. Patients to be tested were asked to sit on a chair, roll up their sleeves above elbow. Venous blood samples were collected using full aseptic measures and samples were immediately transported to Armed Forces institute of Pathology for analysis. Blood samples were collected using full aseptic measures. Samples were transported to lab within one hour of collection while serum was separated within 02 hours. Samples bottles for Mg level were held in vertical position during transport to laboratory. For HBA1C estimation samples were collected in EDTA bottles. Serum magnesium level estimation was done by timed endpoint method using calmagite dye while DXC 600 automated analyzer was used for blood sugar estimation.. All results were verified by Pathologist. A subject with Fasting Plasma Glucose at or above 126 $\mathrm{mg} / \mathrm{dL}(7.0 \mathrm{mmol} / \mathrm{L})$ or random plasma glucose concentration $\geq 200 \mathrm{mg} / \mathrm{dL}(11.1 \mathrm{mmol} / \mathrm{L})$ measured on two separate occasions was considered Diabetic. Hypomagnesemia was considered when Serum magnesium level was less than $0.6 \mathrm{mmol} / \mathrm{L}$ determined by calmagite dye method using auto analyzer. Only previously diagnosed patients of Type 2 diabetes mellitus having age between 40 to 70 years from both gender were selected. Indoor/admitted patients, patients on current nutritional support, malabsorption syndrome, on diuretics, active Tuberculosis infection, CCF, ESRD, CLD and stroke were excluded from study. Quantitative variables included in study were age, duration of diabetes, glycosylated hemoglobin and serum magnesium levels. Qualitative variables used were gender and Hypomagnesemia. All the patients before being sampled were given complete information about the purpose and nature of the study and their consent was obtained. Formal approval of study from hospital ethical committee was also sought. All the data was entered in computer software Statistical Package for Social Sciences (version 16.0). Descriptive statistics were applied out to summarize the data. Mean and standard deviation $( \pm S D)$ was calculated for all the quantitative variables i.e. age, duration of diabetes and serum magnesium levels. Frequency and percentages were calculated for qualitative variables i.e. gender, Hypomagnesemia.

\section{RESULTS}

385 subjects were recruited for study after careful scrutiny using above mentioned inclusion and exclusion criteria. Age range was between 40 to 70 years with Mean age $( \pm \mathrm{SD})$ of $54.99( \pm 7.597)$ years. Out of 385 patients $63.9 \%$ were males (246 out of 385 ) whereas $36.1 \%$ were females (139 out of $385)$. Mean duration of diabetes ( \pm SD) was $6.30( \pm$ $3.661)$ years. Mean serum magnesium levels $( \pm S D)$ 


\begin{tabular}{|c|c|c|c|c|c|}
\hline & N & Minimum & Maximum & Mean & Std. Deviation \\
\hline Age in years & 385 & 40 & 70 & 54.99 & 7.597 \\
\hline \multicolumn{2}{|c|}{ Table-I. Mean age \pm standard deviation of subjects } \\
\end{tabular}

\begin{tabular}{|c|c|c|c|c|c|}
\hline & N & Minimum & Maximum & Mean & Std. Deviation \\
\hline $\begin{array}{c}\text { Duration of type 2 } \\
\text { DM in years }\end{array}$ & 385 & 1 & 22 & 6.30 & 3.661 \\
\hline & \multicolumn{2}{|c|}{ Table-II. Duration of type 2 diabetes mellitus \pm standard deviation of subjects } \\
\hline
\end{tabular}

\begin{tabular}{|c|c|c|c|c|}
\hline Hypomagnesemia & Frequency & Percent & Valid percent & Cumulative percent \\
\hline Yes & 124 & 32.2 & 32.2 & 32.2 \\
\hline No & 261 & 67.8 & 67.8 & 100.0 \\
\hline Total & 385 & 100.0 & 100.0 & \\
\hline
\end{tabular}

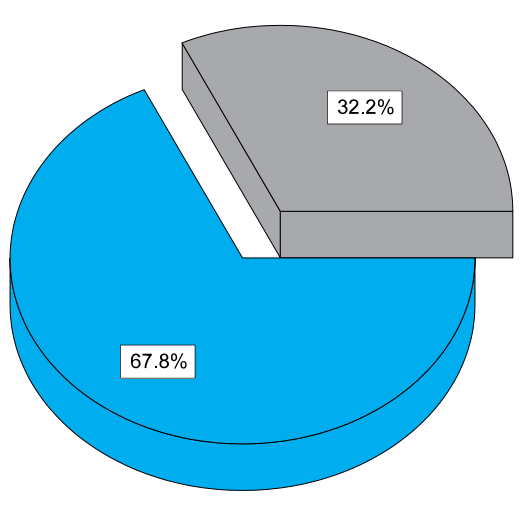

Hypomagnesemia

Normal Mg levels

were $.652( \pm .2130) \mathrm{mmol} / \mathrm{l}$. The overall frequency of Hypomagnesemia was $32.2 \%$ (124 out of the 385 subjects) using the cut-off value of less than $0.6 \mathrm{mmol} / \mathrm{l}$ for Hypomagnesemia, whereas $67.8 \%$ (261 out of the 385 individual) had normal serum magnesium levels.

\section{DISCUSSION}

Hypomagnesemia is present in 13.5 to $47.7 \%$ of patients with type 2 diabetes mellitus (DM) as compared with 2.5 to $15 \%$ of patients without diabetes mellitus. Different studies have suggested that decrease in serum magnesium levels increases the risk of developing type 2 diabetes mellitus in susceptible persons. Hypomagnesemia is also linked to increased risk of diabetic complications. The magnesium deficiency has been associated with chronic diseases, amongst them; diabetes mellitus is one of the most common and important ${ }^{1,4,9.11}$.

Hypomagnesemia is frequently present in diabetic patients, however there is not an exact elucidation of the mechanism of magnesium deficiency in diabetes mellitus. On the other hand, Hypomagnesemia is directly related with some micro and macro vascular complications observed in diabetes, as cardiovascular disease, retinopathy and neuropathy ${ }^{12,17}$. This way, the chronic complications of diabetes can appear precociously. Based on this, the supplementation with magnesium has been suggested in patients with 
diabetes mellitus who have proven Hypomagnesemia and the presence of its complications ${ }^{13}$.Our study revealed that the overall frequency of Hypomagnesemia was $32.2 \%$ (124 out of the 385 subjects) which is quite substantial. Our study lacks the assessment of correlation of Hypomagnesemia and diabetic complications, and effect of magnesium replacement on diabetic control. A study done in Brazil has shown that Hypomagnesemia causes rapid progression to diabetic complications and oral magnesium replacement is associated with better Glycemic control ${ }^{11}$. Moreover it is emphasized that our data cannot be easily applied to whole of population as we focused our study in a highly selected group of type 2 diabetics. Nevertheless, we believe that the study population is a representative sample of the general population of type 2 diabetics in Pakistan ${ }^{2}$. The tested population came from all parts of Pakistan (urban, semi-urban, and rural) and from all social strata. Magnesium is an important intracellular cation. 1 It is often labeled as orphan ion or forgotten ion because not much importance is given to magnesium ion by clinicians despite its role and association with different clinical conditions, diabetes mellitus being one of the most important ${ }^{1,7,9,14}$. One of the objectives of this study was to show that a significant population of diabetics in our country is having Hypomagnesemia. Recently there is a growing interest in clinicians about role of magnesium and different studies have been done. Frequency of Hypomagnesemia was found to be 23 percent in a study by Pham PC et al ${ }^{11}$. It was found to be 29 percent in another study done in America ${ }^{10}$. Studies have also shown that this Hypomagnesemia can accelerate development of diabetic micro and macro vascular complications ${ }^{14,15}$. Liou $\mathrm{K}$ et al have also shown that low plasma magnesium concentrations may contribute to insulin resistance ${ }^{16}$. The relationship between intracellular magnesium and insulin action is also supported by negative correlation between the integrated insulin response after glucose loading and erythrocyte-free magnesium concentration. The results of the prospective study of Sloan F, provided the support for an association between plasma concentration and development of progression of retinopathy in insulin using patients; but whether the plasma magnesium concentration is a causative factor or a marker remains to be determined. In pregnant women with IDDM who are magnesium deficient, lack of magnesium may even account for the high rate of spontaneous abortion and birth defects associated with IDDM ${ }^{17}$. A study to explore further the link between magnesium deficiency and insulin resistance, hypertension and cardiovascular disease looked at the effect of diet lacking in magnesium (< $0.5 \mathrm{mmol} /$ day) on insulin resistance in non-diabetics. The result showed that diet-induced magnesium deficiency leads to decreased insulin sensitivity in lean non-diabetics ${ }^{18}$. Double-blind research indicates that supplementing with magnesium overcomes this problem. Magnesium leads to improved insulin production in elderly people with type $2 \mathrm{DM}^{19}$. In a double-blind, randomized crossover study, Paolisso and colleagues investigated the effect of magnesium supplementation in elderly subjects with insulin resistance on the handling of glucose following an intravenous glucose load and a euglycemic hyperinsulinemic clamp procedure. Magnesium pidolate at $4.5 \mathrm{~g}$ per day (15.8 $\mathrm{mmol} /$ day) for four weeks significantly improved insulin action, oxidativeglucose metabolism, increased erythrocyte magnesium concentration and decreased erythrocyte membrane microviscosity ${ }^{20}$. There are few studies in international literature to assess relation of Hypomagnesemia and diabetes mellitus but no study has been done yet in Pakistan. In majority of studies mentioned above indoor patients were recruited. hospitalized patients can have Hypomagnesemia due to a number of conditions as mentioned above and it is quite hard to exclude influence of all of them. We included only outdoor patients in our study to reduce this bias.

The result of our study when analyzed in context of these studies implies a significant number of diabetics 
having Hypomagnesemia. It is required that studies more studies should be performed to assess effect of magnesium replacement on diabetic complications. No studies have yet compared effect of magnesium replacement in pre diabetics. Studies should also be done to assess whether magnesium replacement can delay onset of overt diabetes mellitus in these patients. In spite of all research, so far mechanism of Hypomagnesemia in diabetics is not known. More research is required to find out mechanism of Hypomagnesemia in diabetics so that corrective measures to prevent these losses can be taken.

\section{CONCLUSIONS}

Significant number of patients with type 2 diabetes mellitus has Hypomagnesemia. It is recommended that serum magnesium levels should be checked in all patients of type 2 diabetes mellitus and magnesium replacement should be done where ever required.

\section{Copyright(c) 07 May, 2013.}

\section{REFERENCES}

1. Bo S, Pisu E. Role of dietary magnesium in cardiovascular disease prevention, insulin sensitivity and diabetes. Curr Opin Lipidol 2008;19:50-6.

2. Bhutto A, Mastoi A, Memon S. Magnesium and its essential role in health. J LUMS 2005;25:56-9.

3. Lee CT, Lien H, Lai LW, Chen JB, Lin CR, Chen HC. Increased renal calcium and magnesium transporter abundance in streptozotocin induced diabetes mellitus. Kidney Int 2006; 69:1786-91.

4. Nathan DM, Buse JB, Davidson MB. Medical Management of Hyperglycemia in Type 2 Diabetes: A Consensus Algorithm for the Initiation and Adjustment of Therapy: A consensus statement of the American Diabetes Association and the European Association for the Study of Diabetes. Diabetes Care 2009;32:193-97.

5. Lima L, Cruz T, Rodrigues LE. Serum and Intracellular magnesium deficiency in patients with metabolic syndrome. Diabetes Res Clin Pract 2009; 83(2):257-
62.

6. Larsson SC, Wolk A. Magnesium intake and risk of type 2 diabetes: a meta-analysis. J Intern Med 2007;262:208-14.

7. Bennett CM, Guo M, Dharmage C. HbA1c as a screening tool for detection of Type 2 diabetes: a systematic review. Diabet Med 2007; 24:333-40.

8. Torii S, Kobayashi K, Takahashi M. Magnesium deficiency causes loss of response to intermittent hypoxia in paraganglion cells. J Biol Chem $2009 \mathrm{Jul}$; 10:179-89.

9. Villegas R, Gao YT, Dai Q, Yang G, Cai H, Zheng W, et al. Dietary calcium and magnesium intake and risk of type 2 diabetes mellitus: the shanghai women's health study. Am J Clin Nutr 2009;89:1059-67.

10. Boulton AJ, Vinik Al, Arezzo JC. American Diabetes Association. Diabetic neuropathies: A statement by the American Diabetes Association. Diabetes Care 2008;28:956-62.

11. Naureen A, Farkhanda $\mathrm{H}$, Tahir M. Familial Hypomagnesemia and hypercalciuria (Manz Syndrome). J Coll Physicians Surg Pak 2006 Jun;16:428-30.

12. Sales CR, De Fatima Campos, Pedrosa L. Magnesium and diabetes mellitus: Their relation. Clin Nutr 2006;25: 554-62.

13. Phuong-Chi T. Pham, Phuong-Mai T. Pham, Son V. Pham, Jeffrey M. Miller, Phuong-Thu T. Pham. Hypomagnesemia in Patients with Type 2 Diabetes. Clin J Am Soc Nephrol 2007;2:366-73.

14. Moles R, Mcmullen W, Rimm E. Magnesium intake and risk of type 2 diabetes in men and women. Diabetes Care 2006;27:134-40.

15. Mizoue T, Yamaji T, Tabata S. Dietary patterns and glucose tolerance abnormalities in Japanese men. $J$ Nutr 2006;136:1352-58.

16. Lindström J, Peltonen M, Eriksson JG. High-fibre, lowfat diet predicts long-term weight loss and decreased type 2 diabetes risk: the Finnish Diabetes Prevention 
Study. Diabetologia 2006;49:912-20.

17. Schulze MB, Hoffmann K, Manson JE. Dietary pattern, inflammation, and incidence of type 2 diabetes in women. Am J Clin Nutr 2005;82:675-84.

18. Liou K, Rodrigues M, Loria M. Magnesium intake and incidence of metabolic syndrome among young adults, Circulation 2006;196: 413-19.
19

19. Costacou T, Ellis D, Fried L, Orchard T. Sequence of progression of albuminuria and decreased GFR in persons with type 1 diabetes: a cohort study. Am J Kidney Dis 2007; 50:721-23.

20. Levitzky Y, Pencina J, D'Agostino B. Impact of impaired fasting glucose on cardiovascular disease: the Framingham Heart Study. J Am Coll Cardiol 2008; 51:264-70.

\section{AUTHOR(S):}

1. DR. RAHEEL IFTIKHAR MBBS, FCPS Medicine, $\mathrm{CMH}$, Kharian

2. DR. SULTAN MEHMOOD KAMRAN MBBS, FCPS Medicine $\mathrm{CMH}$, Kharian.

3. DR. KUMAIL ABBASS, MBBS

Registrar Medicine, $\mathrm{CMH}$, Multan

4. Dr. Ehtesham Haider, MBBS

Registrar Medicine, $\mathrm{CMH}$, Kharian.

\section{Correspondence Address:}

Maj. Dr. Sultan Mehmood

H No. 26/A Shami Road, Okara Cantt. sultanmajoka79@hotmail.com

\section{PREVIOUS RELATED STUDIES}

Mukhtar Ahmad, Khemomal Kharira, Zia ur Rehman. ISCHEMIC HEART DISEASE EVENTS IN DIABETIC PATIENTS HAVING HYPOMAGNESEMIA (Original) Prof Med Jour 10(3) 220 - 222 Jul, Aug, Sep, 2003.

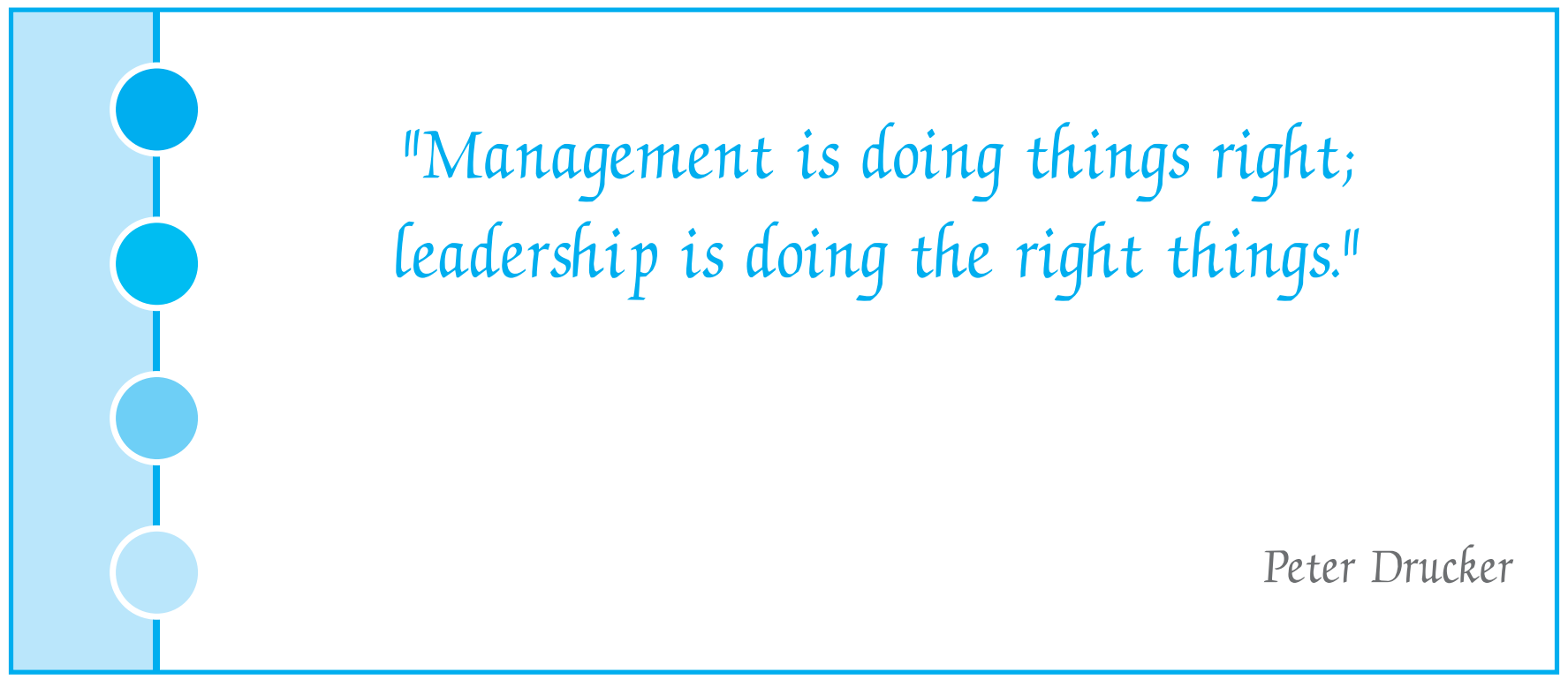

